\title{
Extratos aquosos de frutos verdes de Melia azedarach L. var. azedarach: Investigação da presença de cianeto e avaliação toxicológica
}

\author{
Rita de Cássia A. S. Seffrin ${ }^{1}$ \\ Ervandil Corrêa Costa ${ }^{1}$ \\ Lysandro Borges ${ }^{2}$ \\ Vanessa Corralo Borges ${ }^{2}$ \\ Paulo Cícero do Nascimento ${ }^{3}$ \\ Sônia Thereza Bastos Dequech ${ }^{4 *}$ \\ Carla Daniele Sausen 5

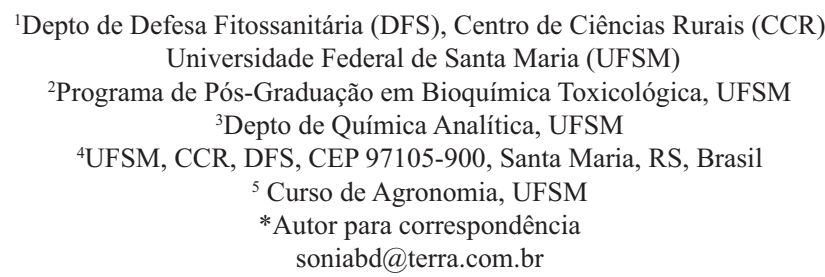

Submetido em 24/10/2007

Aceito para publicação em 14/05/2008

\section{Resumo}

Melia azedarach L., cinamomo, tem sido estudada pela sua ação inseticida e medicinal. Contudo, é uma espécie conhecida pela sua capacidade de produzir compostos cianogênicos e pela presença de saponinas e de compostos polares em frutos verdes, podendo causar intoxicação em animais domésticos e em seres humanos. O presente trabalho teve como objetivos a verificação da presença de cianeto em frutos verdes de $M$. azedarach var. azedarach, espécie adaptada à região central do estado do Rio Grande do Sul, e a avaliação toxicológica, em ratos, de extratos aquosos dessas estruturas vegetais. Os frutos foram colhidos em março de 2005, em área da Universidade Federal de Santa Maria. A presença de cianeto foi avaliada através de medidas voltamétricas e a toxicidade foi analisada através de avaliação da mortalidade, de alterações comportamentais e das funções hepática e renal em ratos Wistar. Os extratos foram administrados através das vias oral e intraperitoneal, nas doses de 2,5, 5,0 e 10\% (p/v, $1 \mathrm{~mL} / \mathrm{kg})$. Pôde-se concluir que frutos verdes de cinamomo não apresentam cianeto em sua composição e extratos aquosos, nas doses analisadas, não causam sintomas de toxicidade em ratos.

Unitermos: toxicidade, inseticidas botânicos, Meliaceae

\section{Abstract}

Aqueous extracts of unripe fruits of Melia azedarach L. var. azedarach: Investigation of cyanide presence and toxicological evaluation. Melia azedarach L., chinaberry, has been studied for its insecticidal and medicinal action. Moreover, it is a species which is known for its capacity of producing cyanogenic composts and for the presence of saponines and polar compounds in unripe fruits, that could be toxic in domestic 
animals and human individuals. The goal of this work was to determine the presence of cyanide in unripe fruits of M. azedarach var. azedarach, a species adapted to the central region of Rio Grande do Sul state, and to carry out a toxicological evaluation, in rats, of aqueous extracts of these plant structures. Unripe fruits were harvested in March 2005, in the area of the Federal University of Santa Maria. The presence of cyanide was evaluated through voltammetric measurements and the toxicity was analyzed through the evaluation of mortality, behavioral changes, and the hepatic and renal functions of Wistar rats. The extracts were administered by oral and intraperitoneal route, at doses of $2.5,5.0$ and $10.0 \%(\mathrm{w} / \mathrm{v}, 1 \mathrm{~mL} / \mathrm{kg})$. It was concluded that unripe fruits of chinaberry did not present cyanide in their composition and that the aqueous extracts, in the analyzed doses, did not cause symptoms of toxicity in rats.

Key words: toxicity, botanical insecticides, Meliaceae

\section{Introdução}

Inseticidas botânicos são compostos resultantes do metabolismo secundário das plantas. As Angiospermae têm, normalmente, pelo menos um tipo de composto secundário em concentração suficiente para reduzir o ataque pelos insetos (Harbone, 1978, apud Menezes, 2005).

Na busca de espécies com ação no controle de pragas, a família Meliaceae foi identificada como um dos grupos mais promissores, uma vez que grande parte de suas espécies têm compostos com ação contra insetos e toxicidade geralmente baixa (Martinez, 2002).

Dentre as meliáceas, o cinamomo (Melia azedarach L.) destaca-se como uma das espécies mais estudadas, sendo que extratos preparados a partir de frutos dessa planta mostraram-se eficientes no controle de Spodoptera frugiperda (J.E. Smith, 1797) (Vendramim e Scampini, 1997; Rodríguez e Vendramim, 1998), de Diabrotica speciosa (Germar, 1824) (Ventura e Ito, 2000) e de Liriomyza huidobrensis (Blanchard, 1926) (Banchio et al., 2003), dentre outros.

Os frutos verdes de $M$. azedarach contêm saponinas e um alcalóide, a azaridina ou mangrovina, que é um narcótico com ação sobre o sistema nervoso central (Zwing, 2001). As saponinas são solúveis em água e tóxicas para os peixes (Saito e Luchini, 1998) e aos seres humanos (Vickery e Vickery, 1981). Melia azedarach é citada na literatura como uma espécie que possui a capacidade de produzir compostos cianogênicos (Gibbs, 1974). Experimentos conduzidos na Austrália indicaram que a toxicidade das plantas pode variar de acordo com a área onde crescem e que algumas árvores podem não ser tóxicas (Oelrichs et al., 1985). Contudo, de acordo com estudos realizados por Seigler (1976), muitas plantas de natureza cianogênica são registradas na literatura, porém necessitando de maiores estudos, sendo que M. azedarach foi incluída, pelo autor, dentre essas espécies.

Intoxicações por M. azedarach têm sido observadas em animais domésticos e em humanos devido à ingestão de frutos e de folhas, sendo que as folhas são menos tóxicas do que os frutos. A toxicidade dos frutos está contida na polpa, na casca e na amêndoa (Hurst, 1942; Kingsbury, 1964). Dantas et al. (2000) demonstraram através de estudos fitoquímicos de frutos de cinamomo, a presença de uma maior concentração de compostos polares no fruto, com destaque para taninos e compostos fenólicos.

Além disso, crianças podem morrer após alguns dias se ingerirem de seis a oito frutos de M. azedarach, sendo que os sintomas da intoxicação são: náuseas, vômitos, diarréia, sede, suor, ringir dos dentes, sonolência e convulsão (Kingsbury, 1964; Everist, 1974; Oelrichs et al., 1985). Contudo, estudos realizados na Argentina mostraram que crianças que ingeriram frutos de $M$. azedarach L não se intoxicaram (Hurst, 1942).

A partir do exposto, esta pesquisa teve como objetivo realizar a investigação da presença de cianeto em frutos verdes de M. azedarach L. var. azedarach, através de detecção voltamétrica. Ainda, avaliar a toxicidade de extratos aquosos, à base de frutos verdes de cinamomo, em ratos Wistar machos albinos, para que esses extratos possam ser utilizados no controle de insetos-praga, com maior segurança aos animais domésticos e aos seres humanos expostos aos mesmos. 


\section{Material e Métodos}

Frutos ainda verdes, mas próximos da maturação, de $M$. azedarach var. azedarach, utilizados tanto para a detecção voltamétrica de cianeto quanto para o preparo de extratos aquosos para a avaliação toxicológica, foram colhidos em março de 2005, em área experimental do Departamento de Fitotecnia da Universidade Federal de Santa Maria (UFSM), Santa Maria, RS.

Para preparo dos extratos, seguiu-se a metodologia proposta por Bogorni (2003). Os frutos foram triturados em liquidificador e imersos em água, para extração dos compostos polares, durante 24 horas a uma temperatura de $10^{\circ} \mathrm{C}$. Foram utilizadas concentrações de 2,5, 5,0 e $10,0 \%$ peso/volume (p/v). Antes da utilização do extrato no experimento, realizou-se a filtragem com tecido fino tipo voal para a retirada do material sólido.

O primeiro ensaio, relativo ao teste para a detecção voltamétrica de cianeto, foi conduzido no Laboratório de Eletroanalítica do Departamento de Química Analítica da UFSM.

A detecção de cianeto é fundamentada na produção de uma onda voltamétrica resultante da reação:

$$
2 \mathrm{CN}^{-}+\mathrm{Hg} \leftrightarrow \mathrm{Hg}(\mathrm{CN})_{2}+2 \mathrm{e}^{-}
$$

Esta reação no eletrodo envolve a oxidação do mercúrio, com a formação imediata de um composto entre cianeto e mercúrio no eletrodo (íon solúvel). Como resultado desta reação, uma onda voltamétrica pode ser obtida com uma corrente de difusão proporcional à concentração de cianeto.

Para detectar a presença ou não de cianeto no analito (amostra de frutos verdes de M. azedarach var. azedarach) utilizou-se o processador $693 \mathrm{em}$ combinação com o 694 VA Stand (Metrohm, Herisau, Switzerland) operando com eletrodo de mercúrio no modo de gota pendente (hanging mercury drop electrode - HMDE). O controle positivo do método voltamétrico foi realizado a partir da adição de cianeto diretamente à célula voltamétrica contendo previamente a amostra (método da adição do padrão).

Todos os reagentes utilizados foram produtos de qualidade para análise e a água foi purificada para o preparo das soluções. Para as medidas feitas por voltametria adotou-se um procedimento que consistiu em adicionar $10 \mathrm{~mL}$ de água $+0,1 \mathrm{~mL}$ de solução de eletrólito suporte $\mathrm{NH}_{3} / \mathrm{NH}_{4} \mathrm{Cl}$ e $\mathrm{KCl}$ (íons) $+1 \mathrm{~mL}$ da amostra, e, após, colocou-se a solução na célula do processador voltamétrico, para avaliação da presença ou não de cianeto na amostra (Brainina et al., 2000).

$\mathrm{O}$ analito foi pré-concentrado num potencial de $-800 \mathrm{mV}$ com um tempo de pré-concentração de 180 segundos e intervalo de varredura de $-800 \mathrm{a}-100 \mathrm{mV}$. O limite de determinação do método situa-se em torno de $1,0 \mu \mathrm{g} / \mathrm{L}$ de cianeto. Desta forma, mesmo considerando o fator de diluição utilizado nas amostras, o cianeto presente pode ser detectado.

Nos ensaios de avaliação toxicológica, foram utilizados ratos Wistar machos albinos, provenientes do Biotério Central da UFSM, de acordo com os procedimentos preconizados pelo Comitê de Ética da Universidade (projeto registrado sob $n^{\circ} 016735$, no GAP/CCR). Os testes foram realizados no Laboratório de Síntese, Reativação e Avaliação Farmacológica e Toxicológica de Organocalcogênios do Departamento de Química da UFSM.

Os animais receberam uma única administração de extrato aquoso de frutos verdes de M. azedarach var azedarach, através da via oral ou intraperitoneal, em diferentes concentrações $(2,5,5,0$ e 10,0\% p/v). A dose com concentração de $10,0 \% \mathrm{p} / \mathrm{v}$ corresponde ao valor máximo normalmente utilizado em testes com extratos de plantas inseticidas, em função da dificuldade, por parte do produtor, em preparar doses com concentrações maiores à citada. Os animais controle receberam somente água destilada $(1 \mathrm{~mL} / \mathrm{kg})$. Cada tratamento foi avaliado em 16 animais, sendo que os animais controle receberam somente água destilada $(1 \mathrm{~mL} / \mathrm{kg})$.

Após a administração dos extratos, os animas foram observados por uma hora, para avaliação de possíveis alterações comportamentais. Decorrido este período, os animais foram colocados em uma sala de criação a $22^{\circ} \mathrm{C}$ com ciclo claro/escuro de 12 horas, com acesso livre à alimentação e água. A sobrevivência foi avaliada por um período de 72 horas, e, após isso, os animais foram eutanaziados e as dosagens bioquímicas foram realizadas em amostras de sangue obtidas através de 
punção cardíaca. Esse tempo de observação está baseado em outros trabalhos de toxicidade, como Meotti et al. (2003) e Savegnago et al. (2006 a e b).

A função hepática foi avaliada através da dosagem da atividade das enzimas aspartato aminotransferase (AST) e alanina aminotransferase (ALT). As enzimas aspartato aminotransferase (AST) e alanina aminotransferase (ALT) foram usadas como marcadores bioquímicos de dano hepático agudo e foram determinados segundo o método de Reitman e Frankel (1957) através de kit LABTEST Diagnóstica S.A., Minas Gerais, Brasil.

A função renal foi analisada pela determinação dos níveis plasmáticos de uréia e creatina (kit LABTEST Diagnóstica S.A., Minas Gerais, Brasil).

A análise estatística foi realizada através de análise de variância (ANOVA), e as diferenças foram consideradas significativas quando $\mathrm{p}<0,05$.

\section{Resultados e Discussão}

O resultado do teste de voltametria foi representado por um gráfico, o qual apresentou uma linha paralela ao eixo das abcissas, indicando que frutos verdes de $M$. azedarach var. azedarach não apresentam cianeto em sua composição (Figura 1), pois não houve reação no eletrodo, nem formação de uma onda anódica.

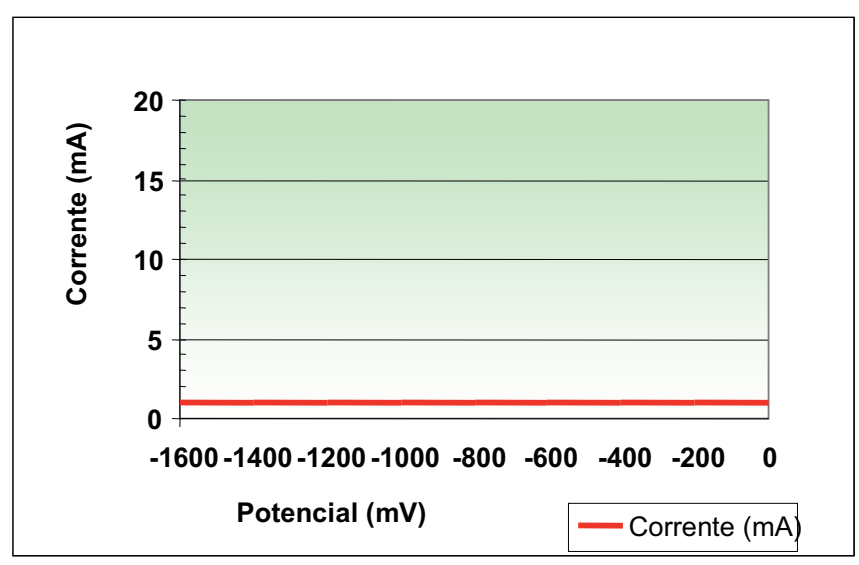

FIGURA 1: Avaliação voltamétrica de cianeto em frutos de Melia azedarach L. var. azedarach colhidos em Santa Maria, RS, 2005.

Quanto à avaliação toxicológica, os tratamentos não provocaram a morte e nem causaram alterações comportamentais nos animais no período de 72 horas, o que equivale à avaliação da toxicidade aguda. Também não houve nenhuma alteração nos níveis plasmáticos das enzimas aspartato aminotransferase (AST) e alanina aminotransferase (ALT) quando comparado aos valores encontrados nos animais controles. A administração dos extratos também não causou nenhuma alteração nos níveis plasmáticos de uréia e creatinina, sugerindo que o extrato de frutos verdes de $M$. azedarach var. azedarach, nas doses testadas, não causou toxicidade renal ou hepática em ratos (Tabela 1).

Entretanto, nos frutos inteiros estão presentes diversos compostos que podem provocar estes sintomas após a ingestão. No extrato aquoso estão presentes somente compostos solúveis em água. Portanto, os compostos polares presentes no fruto, como taninos e compostos fenólicos (Dantas et al., 2000), bem como as saponinas (Saito e Luchini, 1998), não causaram toxicidade quando presentes em extratos aquosos nas doses de 2,5, 5,0 e 10,0\% p/v.

TABELA 1: Análise dos níveis plasmáticos das enzimas aspartato aminotransferase (AST) e alanina aminotransferase (ALT), e dos níveis de uréia e creatinina (média \pm DP de 16 animais por grupo), através de administração oral ou intraperitoneal de extrato aquoso de frutos verdes de Melia azedarach var. azedarach, nas doses de 2,5, 5,0 e 10,0\% $\mathrm{p} / \mathrm{v}$, em ratos.

\begin{tabular}{|c|c|c|c|c|}
\hline \multicolumn{5}{|c|}{ Administração via oral } \\
\hline & AST (U/L) & $\operatorname{ALT}(\mathrm{U} / \mathrm{L})$ & \begin{tabular}{|c|}
$\begin{array}{c}\text { Uréia }(\mathrm{mg} / \\
\mathrm{dL})\end{array}$ \\
\end{tabular} & $\begin{array}{c}\text { Creatinina } \\
(\mathrm{mg} / \mathrm{dL})\end{array}$ \\
\hline Controle & $176,5 \pm 8,59$ & $69,2 \pm 6,8$ & $36,4 \pm 0,6$ & $0,15 \pm 0,03$ \\
\hline $2,5 \%$ & $199,5 \pm 12,6$ & $70,0 \pm 0,8$ & $41,8 \pm 2,2$ & $0,15 \pm 0,03$ \\
\hline $5,0 \%$ & $180,6 \pm 1,9$ & $76,1 \pm 7,5$ & $47,9 \pm 1,1$ & $0,16 \pm 0,04$ \\
\hline $10,0 \%$ & $187,6 \pm 17,4$ & $70,5 \pm 2,9$ & $39,5 \pm 0,5$ & $0,16 \pm 0,04$ \\
\hline \multicolumn{5}{|c|}{ Administração via intraperitoneal } \\
\hline & $\operatorname{AST}(\mathrm{U} / \mathrm{L})$ & $\operatorname{ALT}(\mathrm{U} / \mathrm{L})$ & $\begin{array}{c}\text { Uréia (mg/ } \\
\text { dL) }\end{array}$ & $\begin{array}{c}\text { Creatinina } \\
(\mathrm{mg} / \mathrm{dL})\end{array}$ \\
\hline Controle & $136,2 \pm 3,6$ & $51,7 \pm 3,5$ & $38,6 \pm 0,9$ & $0,14 \pm 0,04$ \\
\hline $2,5 \%$ & $147,5 \pm 15,8$ & $60,5 \pm 3,7$ & $39,0 \pm 8,4$ & $0,13 \pm 0,04$ \\
\hline $5,0 \%$ & $141,4 \pm 15,8$ & $77,3 \pm 13,8$ & $45,9 \pm 1,5$ & $0,13 \pm 0,02$ \\
\hline $10,0 \%$ & $153,1 \pm 11,6$ & $52,5 \pm 0,7$ & $44,8 \pm 1,5$ & $0,12 \pm 0,01$ \\
\hline
\end{tabular}

Os resultados obtidos, no presente trabalho, permitem concluir que extratos aquosos de frutos verdes de $M$. azedarach var. azedarach, colhidos em março 
de 2005, em área experimental do Departamento de Fitotecnia da UFSM, Santa Maria, RS, não apresentam cianeto em sua composição e não causam toxicidade, nas doses de 2,5, 5,0 e 10,0\% p/v, em ratos.

\section{Referências}

Bogorni, P. C. 2003. Efeito de extratos aquosos de Trichilia spp. sobre o desenvolvimento de Spodoptera frugiperda ( J. E. Smith) em milho. Tese de Doutorado Escola Superior de Agricultura Luiz de Queiroz, Universidade de São Paulo, Brasil, 65pp.

Banchio, E.; Valladares, G.; Defago, M.; Palacios, S.; Carpinella, C. 2003. Effects of Melia azedarach (Meliaceae) fruit extracts on the leafminer Liriomyza huidobrensis (Diptera, Agromyzidae). Annual Applied Biology, 143:187-193.

Brainina, K. Z.; Malakhova, N. A.; Stojko, N. Y. 2000. Stripping voltammetry in environmental and food analysis. Fresenius' Journal of Analytical Chemistry, 368 (4): 307-325.

Dantas, D. A.; Maganha, M.; Beretta, T. E.; Pereira, G. S.; Nozu, P.; Matias, R.; Solon, S.; Resende, U. M.; Koller, W. W.; Gomes, A. 2000. Estudo fitoquímico dos frutos de Melia azedarach L. (Cinamomo, Meliaceae). Anais do Encontro de Pesquisa e Iniciação Científica da UNIDERP, Campo Grande, Brasil, p.119-120.

Everist, S. L. 1974. Poisonous plants of Australia. Angus and Robertson Publishers, Sydney, Australia, 684pp.

Gibbs, R. D. 1974. Chemotaxonomy of flowering plants. 4 vols. McGiil-Queen's University Press, Montreal, Canada, 1980pp.

Hurst, E. 1942. Poisonous plants of New South Wales. Plants Committee, NSW, Sydney, Australia, 342pp.

Kingsbury, J. M. 1964. Poisonous plants of the United States and Canada. Prentice-Hall, Englewood Cliffs, USA, 626pp.

Martinez, S. S. 2002. Outras Meliáceas. In: Martinez, S. S. (ed.). O Nim Azadirachta indica - natureza, usos mútiplos, produção. IAPAR, Londrina, Brasil, 142pp.

Menezes, E. L. A. 2005. Inseticidas botânicos: seus princípios ativos, modo de ação e uso agrícola. (Embrapa Agrobiologia. Documentos, 205). Embrapa Agrobiologia, Seropédica, Brasil, 58pp.

Meotti, F. C.; Borges, V.C.; Zeni, G.; Rocha, J. B. T.; Nogueira, C. W. 2003. Potential renal and hepatic toxicity of diphenyl diselenide, diphenyl ditelluride and Ebselen for rats and mice. Toxicology Letters, 143: 9-16.
Oelrichs, P. B.; Hill M. W.; Vallely P. J.; Macleod, J. K.; Molinski T. F. 1985. The chemistry and pathology of meliatoxins A and B constituents from the fruit of Melia azedarach L. var. australasica. In: Seawright, A.; Hegarty, M. P. \& James, L. F. (eds). Plant Toxicology. Queensland Poisonous Plants Committee, Yeerongpilly, Australia, p.387-394.

Reitman, S.; Frankel, S. 1957. A colorimetric method for the determination of serum glutamic oxalacetic and glutamic pyruvic transaminases. American Journal of Clinical Pathology, 28: 56-63.

Rodrígues, H. C.; Vendramim, J. D. 1998. Uso de indices nutricionales para medir el efecto insectistatico de extractos de meliáceas sobre Spodoptera frugiperda. Menejo Integrado de Plagas, 48: 11-18.

Saito, M. L.; Luchini, F. 1998. Substâncias obtidas de plantas e a procura por praguicidas eficientes e seguros ao meio ambiente. EMBRAPA/CNPMA, Jaguariúna, Brasil, 46pp.

Savegnago, L.; Borges, V. C.; Alves, D.; Jesse, C. R.; Rocha, J. B. T.; Nogueira, C. W. 2006a. Evaluation of Antioxidant Activity and Potential Toxicity of 1-Buthyltelurenyl-2-methylthioheptene. Life Sciences, 79: 1546-1552.

Savegnago, L.; Jesse, C. R.; Moro, A.; Borges, V. C.; Santos, F. W.; Rocha, J. B. T.; Nogueira, C. W. 2006b. Bis selenide alkene derivatives: A class of potential antioxidant and antinociceptive agents. Pharmacology Biochemistry and Behavior, 83: 221-229.

Seigler, D. S. 1976. Plants of Oklahoma and Texas capable of producing cyanogenic compounds. Proceedings of the Oklahoma Academy of Science, 56: 95-100.

Vendramim, J. D.; Scampini, P. J. 1997. Efeito do extrato aquoso de Melia azedarach sobre o desenvolvimento de Spodoptera frugiperda (J. E. Smith) em dois genótipos de milho. Revista de Agricultura, 72 (2): 159-170.

Ventura, M. U.; Ito, M. 2000. Antifeedant activity of Melia azedarach (L.) extracts to Diabrotica speciosa (Genn.) (Coleoptera: Chrysomelidae) beetles. Brazilian Archives of Biology and Technology, 22 (2): 215-219.

Vickery, M. L.; Vickery, B. 1981. Secondary plant metabolism. v.1. The Macmillan Press Ltd., London, UK, p.20-55.

Zwing, R. 2001. Plantas tóxicas no jardim e no campo. Disponível em <http: proteus.limeira.com.br/portalverde/notícias $>$. Acesso em 01 de julho de 2005. 